\title{
Magnetic Nanoparticles of Chitosan for Targeted Delivery System of Plasmids to the Lungs
}

\author{
Cynthia Aracely Alvizo Báez, Itza Eloisa Luna Cruz, \\ Maria Cristina Rodríguez Padilla, and Juan Manuel Alcocer González
}

Laboratorio de Inmunología y Virología, Facultad de Ciencias Biológicas, Universidad Autónoma de Nuevo León, 66450 San Nicolás de los Garza, Mexico

Correspondence should be addressed to Cynthia Aracely Alvizo Báez; cynthiabaez@hotmail.com

Received 24 October 2013; Revised 23 January 2014; Accepted 2 February 2014; Published 12 March 2014

Academic Editor: Paresh Chandra Ray

Copyright (C) 2014 Cynthia Aracely Alvizo Báez et al. This is an open access article distributed under the Creative Commons Attribution License, which permits unrestricted use, distribution, and reproduction in any medium, provided the original work is properly cited.

\begin{abstract}
One of the major problems of gene therapy is the efficient, specific, and targeted delivery as well as the safety of the materials used in such systems. The specific targeted delivery of genes to the lung offers the possibility to treat a variety of specific diseases. We developed chitosan nanoparticles with the plasmid pCEM-Luc, which contains a promoter activated by magnetic field. Nanoparticles of 200-250 nm obtained by ionic gelation with a 99\% retention rate were transfected in B16F10 cells and in vivo in the lungs of Balb/c mice by intratracheal administration. We observed that an external magnetic field increased the expression of the luciferase reporter gene in B16F10 cells transfected with magnetic nanoparticles and in homogenized lungs of mice which determined differences in levels of expression between different regions of the lungs (apical or distal and left or right). The highest levels of luciferase activity were observed in the apical left region. The magnetic nanoparticles prove an efficient delivery system to in vitro transfection of cells and lung tissue.
\end{abstract}

\section{Introduction}

Lung gene therapy has attracted remarkable scientific and biomedical interest in recent years for the treatment of a variety of pathologies including cancer that causes around 1.3 million of deaths every year; this is due to a lack of a successful therapy. Particularly important efforts have been made in obtaining systems possessing cell-type specificity for transgene delivery and regulation of its expression by small molecules. For this purpose magnetic nanoparticles have several advantages such as particle size and large surface area which can be properly modified to attach with biological agents, in addition having magnetic response so that they can be manipulated by an external magnetic field gradient; this technique is called magnetofection based on the principles developed by Widder in the $70 \mathrm{~s}$ and its principle is to couple genetic material to nanoparticles; the efficacy has been demonstrated in a variety of cells [1].

In vivo magnetic fields are focused under the site required to promote the transfection and also to carry the therapeutic gene to an organ or a specific site within the body, offering the possibility to develop more efficient and less invasive therapy.

Chitosan nanoparticles are an attractive pharmaceutical delivery vehicle, because they have the capacity to protect sensitive bioactive macromolecules from enzymatic and chemical degradation in vivo and during storage [2].

Chitosan is a biodegradable and biocompatible polysaccharide derived from crustacean shells and can form complexes with anionic macromolecules to yield nanoparticles [3].

A carrier system with a specific amino group, density, or surface properties to deliver drugs plays an important role in increasing the therapy following these mechanisms: (a) the protein of interest can be directed at the action site without affecting healthy tissue, (b) the protein of interest could be protected from degradation and remain stable, and (c) the protein of interest could be capable of prolonging the drug at the desired site.

The aim of this work was to develop and prove the efficacy of a gene delivery system targeted to the lungs, using chitosan 
nanoparticles as a vehicle, to enable expression of a luciferase reporter gene reporter using a magnetic field.

\section{Material and Methods}

\subsection{Materials}

2.1.1. Chemicals. Chitosan $(25 \mathrm{kDa})$ was purchased from Coyotefoods Bropolymer and Biotechnology, México. Cell culture media, fetal bovine serum, and cell culture supplements were obtained from GIBCO (Grand Island, NY, USA). Tripolyphosphate (TPP) was obtained from SIGMA (St Louis, USA). Magnetic nanoparticles were obtained CombiMag (OZ Biosciences, Marseille, France). Luciferase Assay System was obtained from Promega (Madison, WI, USA). Plasmid purification kit was obtained from Invitrogen (San Diego, CA, USA).

2.1.2. Plasmid. pCEM-Luc of 5000 bp length, composed of luciferase gene under the control of hsp70 promoter which contains elements of response to CEM (electromagnetic field), was obtained and characterized in our laboratory [4].

\subsection{Methods}

2.2.1. Production of Nanoparticles of Chitosan (CS-NPs). CSMNPs were formulated using the ionotropic gelation between positively charged chitosan and negatively charged TPP as first reported by Calvo et al., 1997 [5]. Chitosan solution was prepared at $2 \mathrm{mg} / \mathrm{mL}$ dissolved in deionized water under stirring. TPP solution $(0.84 \mathrm{mg} / \mathrm{mL})$ containing pDNA (50 and $100 \mu \mathrm{g}$ ) was dropped into the CS solution in equal volume while mixing at $900-1000 \mathrm{rpm} / \mathrm{min}$ at a room temperature (RT). The NP suspension was gently stirred for $30 \mathrm{~min}$ at RT for gelation. To produce the magnetic nanoparticles of chitosan CS-MNPs a solution of CombiMag was added $(1 \mu \mathrm{L} / \mu \mathrm{g}$ of DNA) to the CS-NPs.

2.2.2. Particle Morphology. The morphology and size of NPs were performed by transmission electron microscopy (TEM). One drop of the aqueous dispersion of nanoparticles was added to a coated copper grid, air-dried in vacuum desiccators, and then examined under an electron microscope. Also nanoparticles were analyzed by atomic force microscopy (MFA). For this purpose the samples were diluted with bidistilled water and placed on a microscope slide to analyzate [6].

2.2.3. Zeta Potential. The zeta potential of nanoparticles was measured in a phosphate buffer ( $\mathrm{pH}$ 6) using a zetasizer (Nanotrac U2514ZS) system.

2.2.4. In Vitro Release Studies. Adequate protection of plasmid DNA promoted by its encapsulation in the CS-NPs was performed using an agarose gel tested at different plasmidchitosan ratios $(1: 20,1: 30,1: 40,1: 50$, and $1: 60)$ to choose the best ratio; also plasmid DNA release was determined by incubating the CS-NPs ( $1 \mu \mathrm{g}$ pDNA) in PBS at $37^{\circ} \mathrm{C}$; at appropriate time intervals samples were centrifuged and the supernatant was replaced with fresh medium, and the amount of DNA released in the supernatant was quantified in a nanodrop (Thermo scientific nanodrop 2000).

2.2.5. Encapsulation Efficiency of Nanoparticles. The encapsulation efficiency (EE) was calculated by the DNA content that in entrapped into nanoparticles as shown in the following equation. Encapsulation efficiency $(\%)=$ Actual drug loading/Theoretical drug loading $\times 100 \%$.

2.2.6. In Vitro Transfection Studies in B16F10 Cell Line. Cells were seeded 24 hours (h) prior to transfection into a 24-well plate at a density of $1 \times 10^{4}$ per well in $1 \mathrm{~mL}$ of complete medium (DMEM containing 10\% fetal bovine serum, supplemented with antibiotic antimicotic 1\%) until confluence was attained. CS-NPs (1 $\mu \mathrm{g}$ of DNA for each well) and CS-MNPs were added to the cells, and a magnetic plate was placed under the 24-well plate for 20 minutes and incubated for $24 \mathrm{~h}$ at $37^{\circ} \mathrm{C}$ for luciferase assay. CS-NPs without exposition to a magnetic field were used as a control. The cells were washed twice with ice-cold PBS, were lysated in $100 \mu \mathrm{L}$ of buffer (Promega, Madison, WI, USA), and recovered $20 \mu \mathrm{L}$ of the lysate in a luminometer reader using the luciferase assay kit (Promega, Madison, WI, USA). Luciferase activity in cell lysates was expressed as relative light units (LU/min per $\mathrm{mg}$ of protein in the cell lysate).

2.2.7. In Vivo Studies. 7-week-old adult (25-30 g) male $\mathrm{Balb} / \mathrm{c}$ mice were used for in vivo transfections. Mice were anesthetized intramuscularly with xylazine $(50 \mathrm{mg} / \mathrm{kg})$ and ketamine $(5-10 \mathrm{mg} / \mathrm{kg})$. The trachea was exposed through a skin incision using a sterile surgical set-up and the nanoparticles CS-MNPs (50 $\mu \mathrm{g}$ DNA) were introduced using a $0.5 \mathrm{~mL}$ needle; naked plasmid was used as a control. After transfection immediately a magnet was placed on the thorax with an adhesive for $72 \mathrm{~h}$. Mice without magnet were used as a second control. The animal procedure was approved by the Local Ethics Committee and carried out according to the legal guidelines.

2.3. Detection of Luciferase Activity. Mice were sacrificed by cervical dislocation; lungs were removed, dissected into four regions (apical and distal of the right and left side), and washed with PBS twice homogenized in $200 \mu \mathrm{L}$ of lysis buffer (potassium phosphate, $\mathrm{pH} 7.8$, and $1 \mathrm{mM}$ dithiothreitol (Sigma, St Louis, USA)) using a polypropylene micropestle, followed by 3 cycles of freezing and thawing; then the samples were centrifuged $(16,000 \mathrm{~g})$ at $4^{\circ} \mathrm{C}$ for $4 \mathrm{~min}$ and recovered supernatant. Luciferase activity was assayed with $100 \mu \mathrm{L}$ of lung lysate supernatant and $20 \mu \mathrm{L}$ of the substrate as indicated by the manufacturer. Luciferase activity in the mouse lung was expressed as relative light units/mg lung lysate.

2.3.1. Statistics. Statistical analysis of the data obtained in the evaluation of transfections was determined between groups using ANOVA test and by the Tukey nonparametric test. These analyses were performed using SPSS version 17.0. 


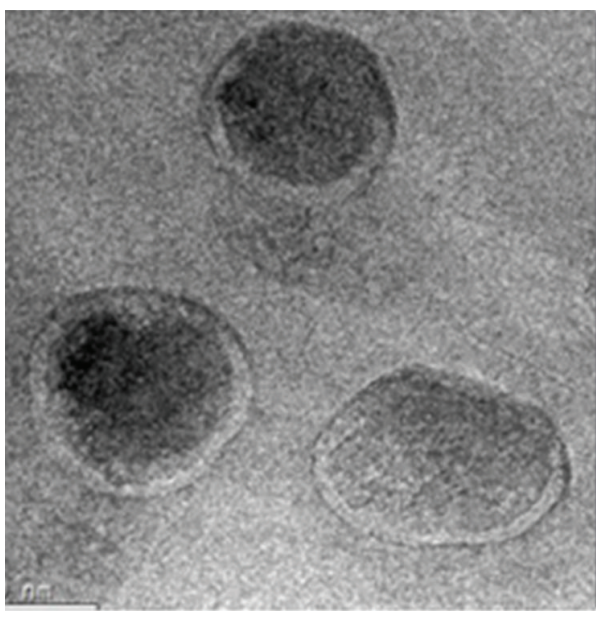

(a)

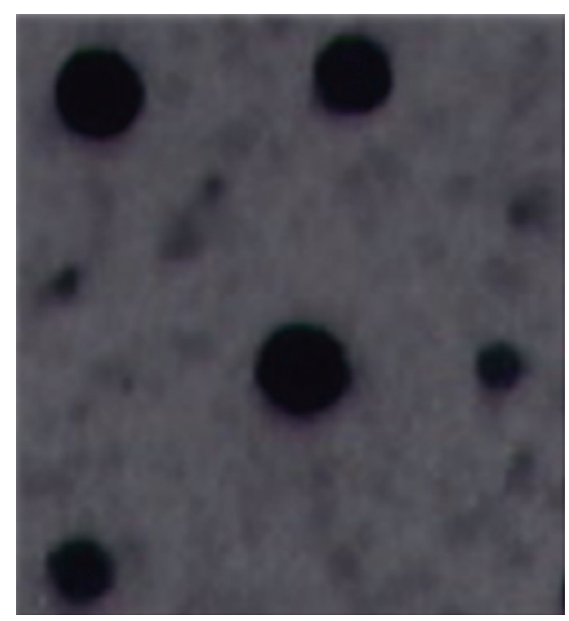

(b)

FiguRE 1: Transmission electron microscopy. Images of nanoparticles with the size of $100-150 \mathrm{~nm}$. (a) Nanoparticles formed by chitosan/plasmid with the size of 100-150 nm (CS-NP). (b) Nanoparticles formed by chitosan/plasmid/magnetic nanoparticle with the size of 200-250 nm (CS-MNP).

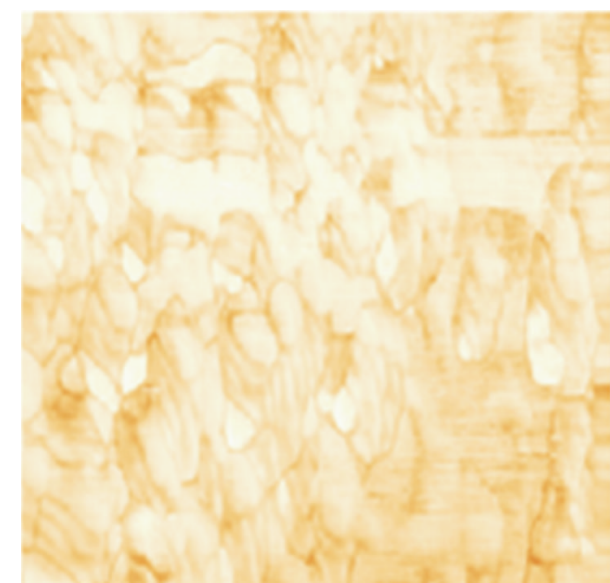

(a)

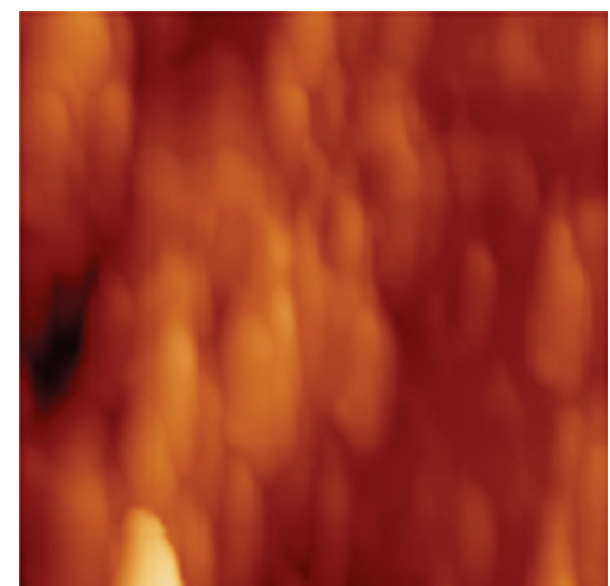

(b)

Figure 2: Atomic force microscopy. Images of nanoparticles. (a) Nanoparticles formed by chitosan/plasmid with the size of 100-150 nm. (b) Nanoparticles formed by chitosan/plasmid/magnetic nanoparticle with the size of $200-250 \mathrm{~nm}$.

\section{Results and Discussion}

3.1. Characterization of Chitosan-DNA Nanoparticles. CSNPs and CS-MNPs were formed bythe ionic gelation technique with the aim of entrapping more plasmid within the nanostructures by extreme encapsulation. In addition TPP was chosen as crosslinking polyanionic agent, due to its unique properties of nontoxicity and ability to instantly form a gel on contact with chitosan. The dropwise addition of a precise amount of TPP into chitosan solution enabled the formation of NPs of comparable size on the nanometer scale. The NPs analyzed by transmission electron microscopy or atomic force microscopy demonstrated a well-defined spherical shape of 150-200 nm appropriated size (Figures 1 and 2 ); this is important as it has previously been demonstrated that the shape of NPs influences the efficiency of transfection.
Spherical NPs lead to better entrapment than nanoparticles with a different morphology. This interesting phenomenon is related to the time taken for a cell to entrap particles with different shapes, which varies in accordance with the volume ratio of the NPs.

3.2. Zeta Potential. The electrostatic potential that exists at the shear plane of a particle, which is related to both surface charge and the local environment of the particle (the zeta $(\xi)$ potential) [7] in our samples, was of $-25 \mathrm{mV}$ with a negative polarity.

3.3. In Vitro Release of pDNA. Developments of NPs that are stable under extracellular environment to protect the genetic material from serum nucleases are a major concern, which could affect the DNA stability and consequently the 


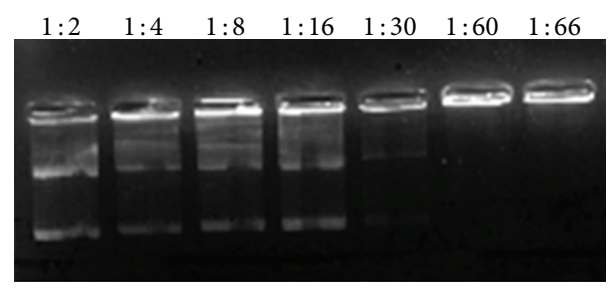

FIgURE 3: Agarose gel. DNA release from chitosan-plasmid complexes at different ratios.

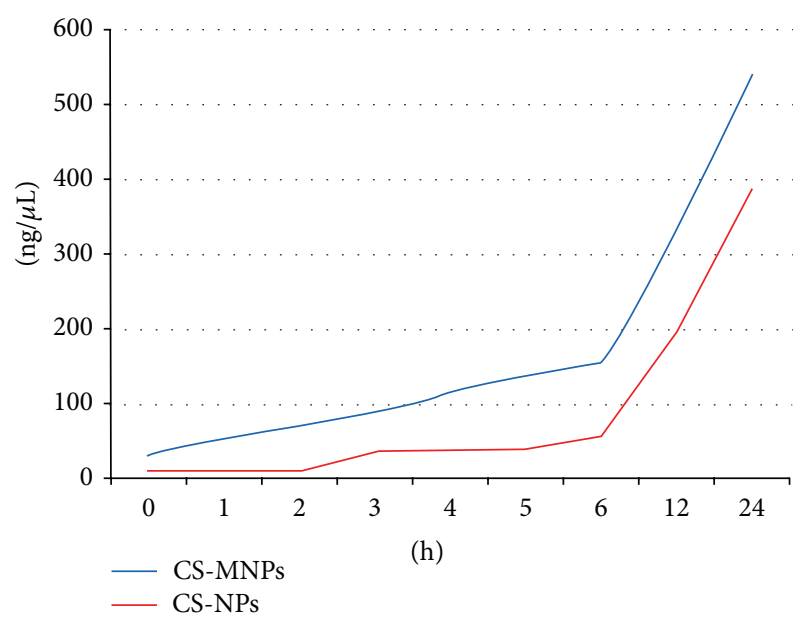

FIGURE 4: Graphical representation of released plasmid pCEMLuc in hours. Magnetic nanoparticles of chitosan (CS-MNPs) and chitosan nanoparticles (CS-NPs).

transfection efficiency. On an agarose gel different plasmidchitosan ratios were tested and it was determined that the best ratio was 1:30 to perform the NP as shown in Figure 3. The release of pDNA efficiency of CS-NPs or CS-MNPs calculated as previously described shows that plasmids were detected at 30 min after formulation of CS-MNPs, but CS-MNPs plasmid was detected $1 \mathrm{~h}$ after formulation (Figure 4 ). The efficiency of encapsulation calculated was $99 \%$.

3.4. In Vitro Transfection Efficiency of Nanoparticles for Gene Expression. The expression of luciferase in B16F10 cells transfected with the CS-NPs exposed to the magnetic field increased statistically $(P=0.05)$ until 20,000 RLU (Relative luciferase units) in comparison with 4,585 RLU obtained in cells unexposed to the magnetic field. This transfection demonstrates that the promoter of the pCEM-Luc plasmid is activated when it is in contact with the magnetic plate. When we transfected the cells with the CS-MNPs exposed to magnetic plate, the levels of gene expression increased from 9,421 RLU to 100,000 RLU compared with control without magnetic field. This magnetic nanoparticle, as gene delivery system induced by magnetic fields, produced the highest expression of luciferase at tenfold (Figure 5).

Although the use of magnetic nanoparticles has been widely used with good results in gene expression $[8,9]$, the

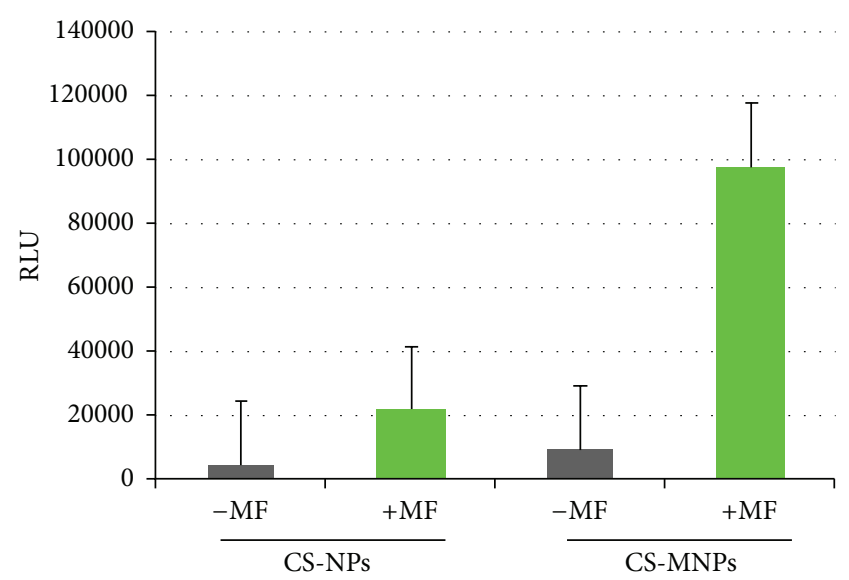

FIgURE 5: Determination of luciferase activity. Expression efficiency in B16F10 cells transfected with CS-NPs or CS-MPs exposed (+MF) or unexposed (-MF) to magnetic field.

TABLE 1: Data averages of the RLU expressed in the four sections of the lungs.

\begin{tabular}{lcccc}
\hline & Distal-L & Apical-L & Distal-R & Apical-R \\
\hline pCEM-Luc - MF & 2,746 & 3,475 & 3,360 & 2,857 \\
pCEM-Luc + MF & 15,054 & 23,598 & 14,259 & 10,479 \\
CS-MNPs + MF & 21,062 & 33,002 & 27,555 & 17,725 \\
\hline
\end{tabular}

use of a plasmid that can be activated by magnetic field shows an increased gene expression significantly.

3.5. In Vivo Transfection Efficiency of Nanoparticles for Gene Expression. For the experiments in vivo, the naked plasmid or CS-MNPs were administrated to mice by via intratracheal which is a fast metohd to deliver particles to the lungs also is secure and allows only the required doses. The lung homogenates of control mice inoculated with pCEM-Luc naked plasmid showed lowest levels of luciferase expression of about 3,000 RLU in the four regions of the lungs; when applying a magnet to mice, the luciferase expression increased statistically $(P=0.05)$. Obtaining a major expression in an apical left region (23,598 RLU) the other three regions, distal left (15,054 RLU), distal right (14,259 RLU), and right apical $(10,479)$, showed similar levels. However the highest levels of activity luciferase compared with the controls $(P=0.05)$ were obtained by the administration of CS-MNPs and magnet exposition on lungs of mice. The levels of luciferase activity were in left apical region of 33,002 RLU following 27,555 RLU in right distal, 21,062 RLU in left distal, and 17,725 RLU in right apical regions (Figure 6). The levels of luciferase activity are resumed in the Table 1 . Those results show the efficiency of magnetic nanoparticles as a gene delivery system, founding the highest activity in the apical left region possibly due to the position of the magnet. 


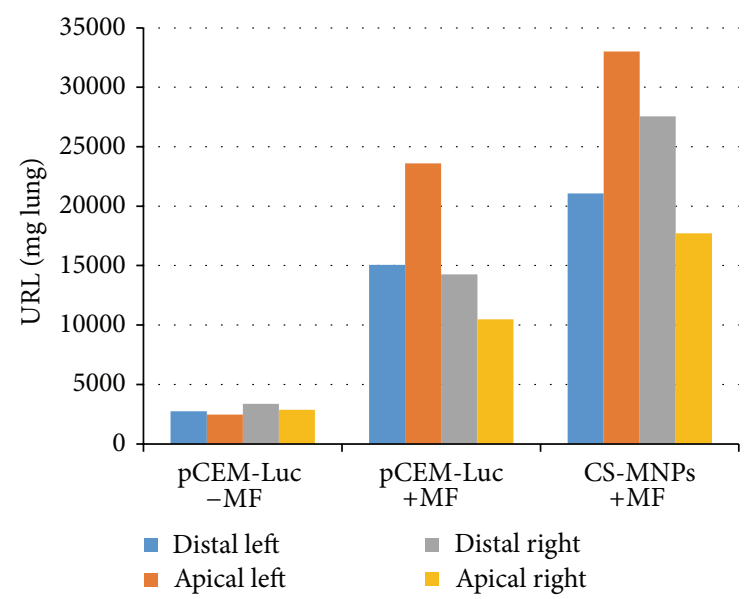

FIGURE 6: Determination of luciferase activity in vivo. Expression of luciferase in different regions of lungs (distal-L, apical-L, distal$\mathrm{R}$, and apical-R) transfected with naked plasmid unexposed to a magnetic field (pCEM-MF) and exposed to a magnetic field (pCEM+MF) or magnetic nanoparticles exposed to a magnetic field (CS-MNPs+MF).

\section{Conclusion}

According to the data obtained in the present investigation the gelation ionic method to produce CS-NPs is easy, fast and can be stable. Nanocarriers based on chitosan produced in this study transfected cells and tissue with good expression of the luciferase gene and even more using the plasmid inducible by the influence of a magnetic external field.

Therefore this investigation represents the experimental support of nanocarriers based on chitosan which are adequate vehicles to deliver and activate genes in the lung tissue under the influence of a magnetic external field. Also this study shows that the promoter in the pCEM-Luc plasmid can be used to manipulate gene expression in lung tissue.

Data shown opens new vision to control specific expression of genes and provide the basis to propose that the nanocarrier system based on chitosan and plasmid with elements of activation under magnetic field could have applications for delivery and remote control of expression of therapeutic genes in a specific tissue.

\section{Conflict of Interests}

The authors declare that they have no conflict of interests.

\section{References}

[1] J. Dobson, "Gene therapy progress and prospects: magnetic nanoparticle-based gene delivery," Gene Therapy, vol. 13, no. 4, pp. 283-287, 2006.

[2] H.-Q. Mao, K. Roy, V. L. Troung-Le et al., "Chitosan-DNA nanoparticles as gene carriers: synthesis, characterization and transfection efficiency," Journal of Controlled Release, vol. 70, no. 3, pp. 399-421, 2001.

[3] D. Kavaz, T. Çirak, E. Öztürk, C. Bayram, and E. B. Denkbaş, "Preparation of magnetic chitosan nanoparticles for diverse biomedical applications," in Functionalized Nanoscale Materials, Devices and Systems, NATO Science for Peace and Security B, pp. 313-320, Springer, Amsterdam, The Netherlands, 2008.

[4] A. O. R. de la Fuente, J. M. Alcocer-González, A. J. HerediaRojas et al., "Effect of $60 \mathrm{~Hz}$ electromagnetic fields on the activity of hsp70 promoter: an in vitro study," Cell Biology International, vol. 33, no. 3, pp. 419-423, 2009.

[5] P. Calvo, C. Remuñan-López, J. L. Vila-Jato, and M. J. Alonso, "Chitosan and chitosan/ethylene oxide-propylene oxide block copolymer nanoparticles as novel carriers for proteins and vaccines," Pharmaceutical Research, vol. 14, no. 10, pp. 1431-1436, 1997.

[6] D. Hritcu, M. I. Popa, N. Popa, V. Badescu, and V. Balan, "Preparation and characterization of magnetic chitosan nanospheres," Turkish Journal of Chemistry, vol. 33, no. 6, pp. 785-796, 2009.

[7] Y. Zhang, M. Yang, N. G. Portney et al., "Zeta potential: a surface electrical characteristic to probe the interaction of nanoparticles with normal and cancer human breast epithelial cells," Biomedical Microdevices, vol. 10, no. 2, pp. 321-328, 2008.

[8] Y. Sun, Z.-L. Chen, X.-X. Yang, P. Huang, X.-P. Zhou, and X.-X. $\mathrm{Du}$, "Magnetic chitosan nanoparticles as a drug delivery system for targeting photodynamic therapy," Nanotechnology, vol. 20, no. 13, Article ID 135102, 2009.

[9] M. Chorny, B. Polyak, I. S. Alferiev, K. Walsh, G. Friedman, and R. J. Levy, "Magnetically driven plasmid DNA delivery with biodegradable polymeric nanoparticles," The FASEB Journal, vol. 21, no. 10, pp. 2510-2519, 2007.

[10] H.-Q. Mao, K. Roy, V. L. Troung-Le et al., "Chitosan-DNA nanoparticlesas gene carriers: synthesis, characterization and transfection efficiency," Journal of Controlled Release, vol. 70, no. 3, pp. 399-421, 2001. 

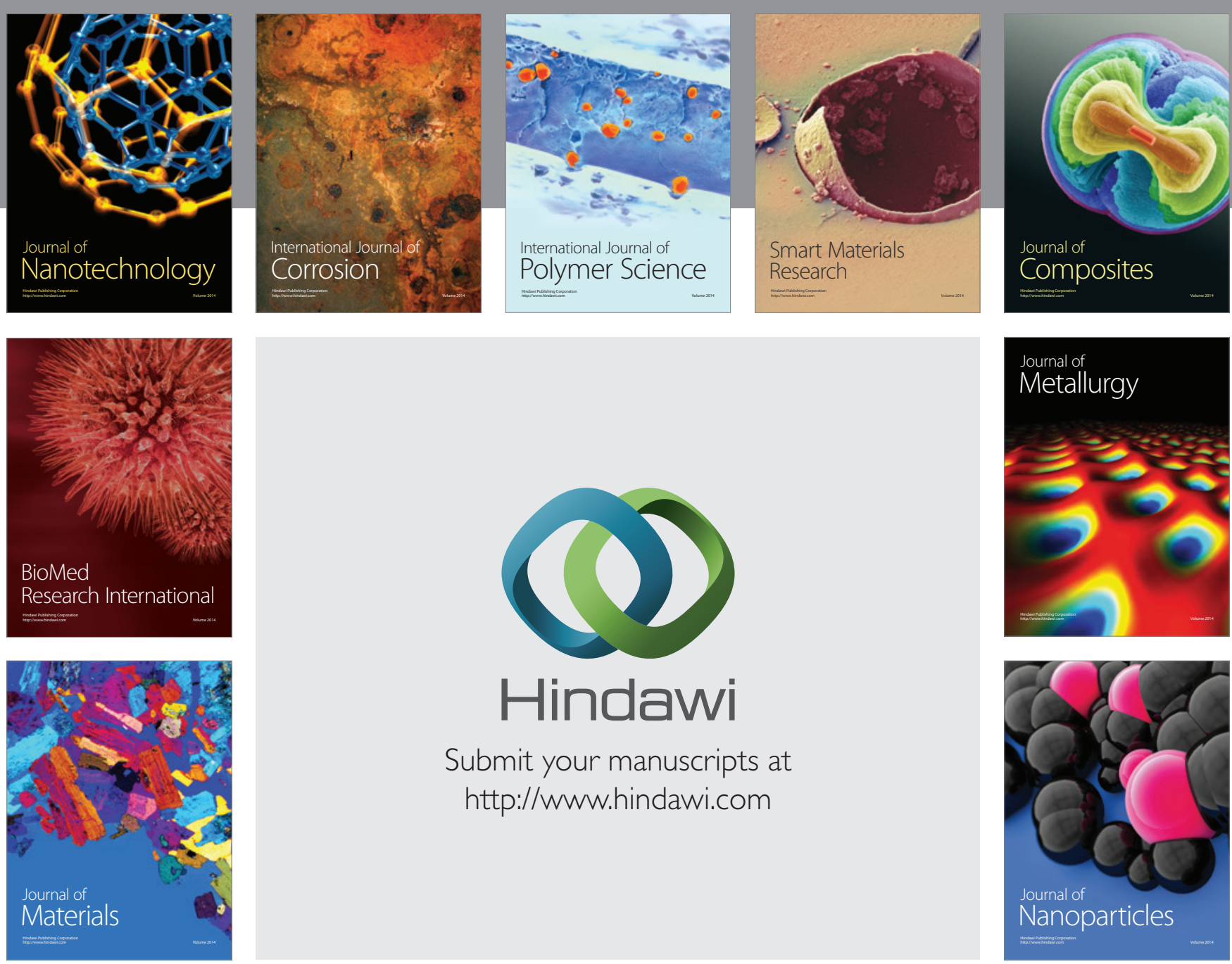

Submit your manuscripts at http://www.hindawi.com
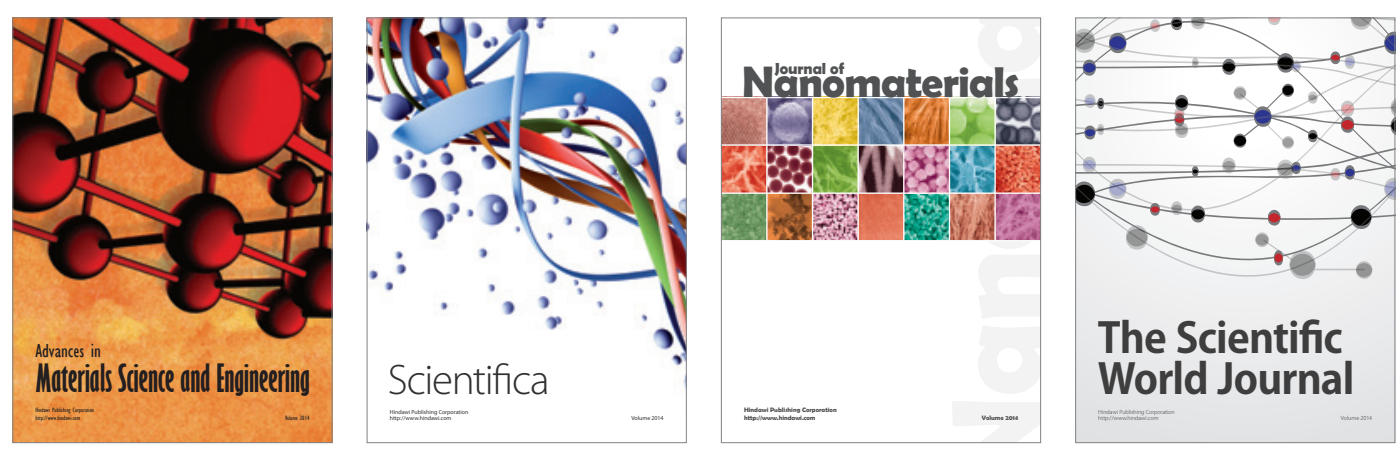

\section{The Scientific World Journal}
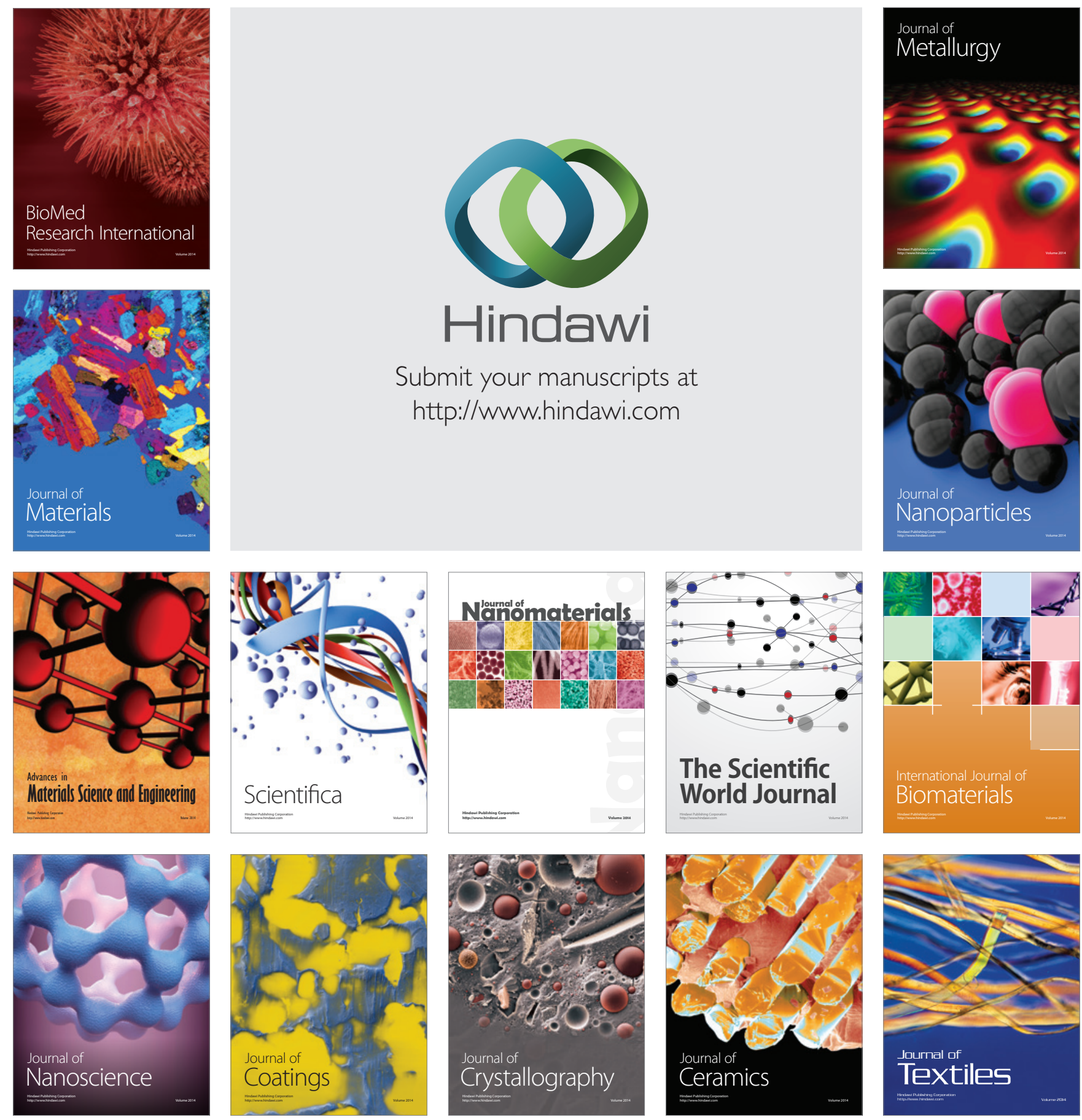